\title{
A NOTE ON THE FLOW INDUCED BY A LINE SINK BENEATH A FREE SURFACE
}

\author{
G. C. HOCKING and L. K. FORBES
}

(Received 17 August 1989; revised 28 March 1990)

\begin{abstract}
The problem of withdrawing water through a line sink from a region containing an homogeneous fluid beneath a free surface is considered. Assuming steady, irrotational flow of an ideal fluid, solutions with low Froude number containing a stagnation point on the free surface above the sink are sought using a series substitution method. The solutions are shown to exist for a value of the Froude number up to a critical value of about 1.4. No solutions of this type are found for Froude numbers greater than this value.
\end{abstract}

\section{Introduction}

This paper considers the steady irrotational withdrawal of a homogeneous incompressible and inviscid fluid through a line sink beneath a free surface. In a fluid of infinite depth, the flow is determined by a single parameter, the Froude number, defined as $F=m /\left(g H^{3}\right)^{1 / 2}$, where $m$ is the strength of the sink, $H$ is the depth of the sink beneath the undisturbed level of the free surface and $g$ is the acceleration caused by gravity.

Solutions have been found previously for the case in which the Froude number is large, and the free surface is drawn down into a cusp shape directly above the sink (see Fig. 1), for many differing geometries. In the case of a fluid of infinite depth, these solutions were found to exist only at a single value of the Froude number ([2], [5], [12]), while in the case of a fluid of finite depth, they were found to exist over a range of values of Froude number between some lower bound (usually unity), and infinity ([3], [6], [7], [9], [13]).

\footnotetext{
'Department of Mathematics, University of Western Australia, Nedlands, Western Australia.

${ }^{2}$ Department of Mathematics, University of Queensland, St. Lucia, Queensland.

(C) Copyright Australian Mathematical Society 1991, Serial-fee code 0334-2700/91
} 


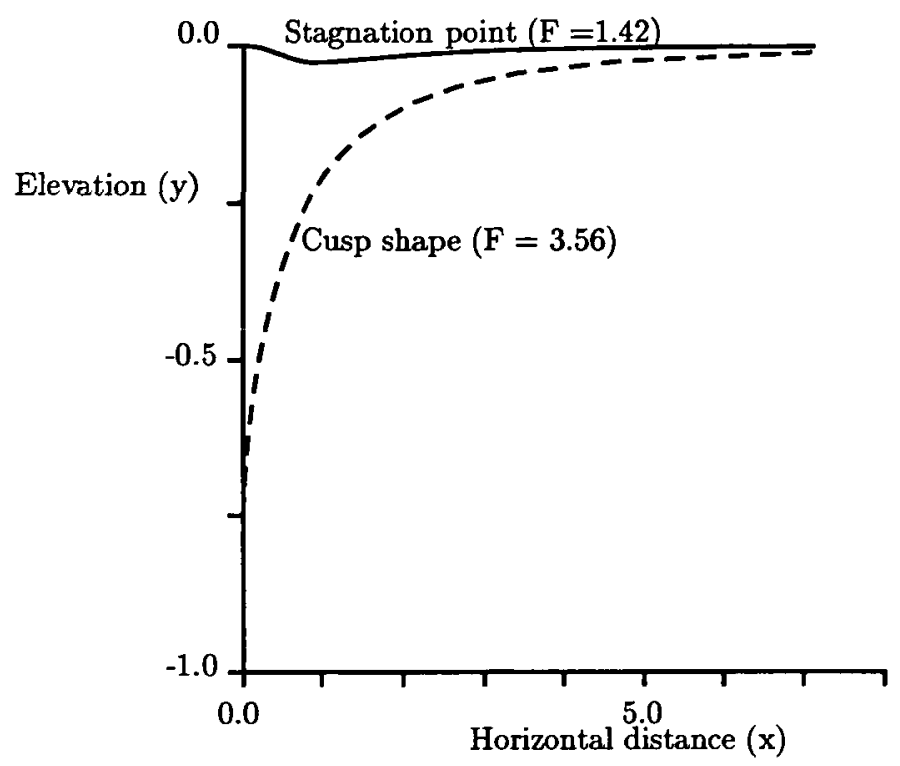

Figure 1. Definition sketch for the problem under consideration. The two possible free surface shapes are shown, a cusp shape and a stagnation point above the sink. These are a comparison of the actual solutions at $F=1.4$ (stagnation point) and $F=3.56$ (cusped shape). The sink is situated at $y=-1.0$ on the scale of this diagram.

For the geometry examined in this paper, a Froude number of 3.56 produced the only cusp-like solution [12].

At low values of Froude number, approximate solutions have been computed which have a stagnation point on the free surface above the sink (see Figure 1). Peregrine [11] computed solutions as a perturbation about the undisturbed level of the free surface for small values of the Froude number. This method developed inconsistencies as $F$ was increased, and in fact the series was found to be divergent, indicating the solutions were only asymptotically valid. Vanden-Broeck, Schwartz and Tuck [14] attempted to sum a divergent series to obtain solutions, but they also encountered difficulties as the value of the Froude number was increased, with a singularity appearing on the free surface.

Tuck and Vanden-Broeck [12] mention briefly that they attempted to compute solutions with a stagnation point using their series method, but that small waves of apparently numerical origin appeared on the computed free surface as $F$ was increased. Their series method was very successful in computing the cusp-like solutions, however, and variations upon it were used successfully on different geometries by other researchers [5], [13].

These results for flows with a stagnation point suggest that there may be some upper bound upon the existence of these solutions as the Froude number 
is increased. In the analogous three-dimensional axisymmetric flow into a point sink, Forbes and Hocking [4] obtained a limiting solution with a Froude number approximately equal to 6.4 , in which a secondary stagnation point formed on the free surface a small distance from the primary stagnation point. No solutions were obtained for larger values of the Froude number.

It is possible to show [12] that the only solutions which can exist to this problem are those with a cusp and those with a stagnation point on the free surface above the sink. In the analogous problem of the flow of two layers of different density, the interface is drawn down directly into the sink, entering at some finite angle (Imberger [8], Wood and Lai [15]), at Froude numbers above a certain critical value.

In the case of a fluid of finite depth, Mekias and Vanden-Broeck [10], have found stagnation-point solutions for Froude numbers greater than a value of about 1.2.

In this paper, the series substitution method of Tuck and Vanden-Broeck [12] is used to compute solutions with a stagnation point on the free surface above the sink in a fluid of infinite depth. The difficulty of spurious small waves on the surface is reduced if enough coefficients in the series are computed, and the results reveal that the waves are a sign of the imminent breakdown of the numerical scheme when more coefficients are used.

Section 2 of this note describes the method of Tuck and Vanden-Broeck, and the results of the numerical method and some discussion are given in Section 3.

\section{Series substitution method}

The series-solution method of Tuck and Vanden-Broeck is described in [12], but for completeness we repeat it below.

We seek a complex potential $f(z)=\phi(x, y)+i \psi(x, y)$ for the steady irrotational flow of an inviscid incompressible fluid of infinite depth, situated beneath a free surface. A line sink of strength $m$ is situated a distance $H$ beneath the free surface. The above assumptions lead to a requirement that the potential satisfies Laplace's equation,

$$
\nabla^{2} \phi(x, y)=0
$$

within the flow domain or equivalently that $f(z)$ be analytic. The condition that the fluid can not cross its own surface leads to the kinematic boundary condition on the free surface, $y=\eta(x)$,

$$
\eta_{x} \phi_{x}-\phi_{y}=0
$$


and the condition that the pressure be constant on the free surface, obtained from the Bernoulli equation, is

$$
g \eta(x)+\left(\phi_{x}^{2}+\phi_{y}^{2}\right) / 2=C,
$$

where $C$ is an arbitrary constant.

If we set the value of the constant in (3) to be zero, the elevation of the free surface will be zero at the stagnation point. At large distances from the sink, the flow will slow to zero, and the free surface will tend toward the stagnation level. The choice of this constant sets the origin of the coordinate system to be at the stagnation level, directly above the sink, and the sink to be situated at $y=-H$.

Defining a length scale $m^{2 / 3} /\left(8 \pi^{2} g\right)^{1 / 3}$, and a velocity scale $(m g / \pi)^{1 / 3}$, (3) becomes

$$
\eta^{\prime}+\left(\phi_{x}^{\prime 2}+\phi_{y}^{\prime 2}\right)=0
$$

where the dashes denote nondimensional variables. Using these variables, the forms of (1) and (2) are unchanged. Henceforth we shall omit the dashes from the nondimensional variables. This choice of scales means that the Froude number is given by

$$
F=\left(8 \pi^{2} / b_{0}^{3}\right)^{1 / 2} \text {, }
$$

where $b_{0}$ is the nondimensional depth of the sink. We define a new complex variable $t$ so that

$$
e^{f}=4 t /(t+1)^{2}
$$

and represent $z=x+i y$ as a series in powers of $t$ of the form

$$
z(t)=\frac{-i}{(t+1)} \sum_{j=0}^{\infty} b_{j} t^{j},
$$

for some real coefficients $b_{j}$ to be determined. The equivalent domains for the $f, z$, and $t$ planes are shown in Figure 2. The flow is symmetric about the line $x=0$.

The choices for (6) and (7) automatically satisfy the flow equations (1) and (2) if the $b_{j}$ are real, and (4) will later be used to obtain the unknown values of $b_{j}, j=0,1,2 \ldots$ Thus as $t \rightarrow 0, z \rightarrow-i b_{0}$ and $f \rightarrow \ln \left(z+i b_{0}\right)$, and represents a sink of strength $2 \pi$ located at $z=-i b_{0}$. As $t \rightarrow-1$, $f \rightarrow 2 \ln z$, which is correct since the compression of the flow beneath the free surface makes the flow look like that of a line sink of strength $4 \pi$ at the origin when viewed from the far field.

The real axis of the $t$-plane is pure imaginary in the $z$-plane, and hence corresponds to a vertical line passing through the sink. The right half of the 
a

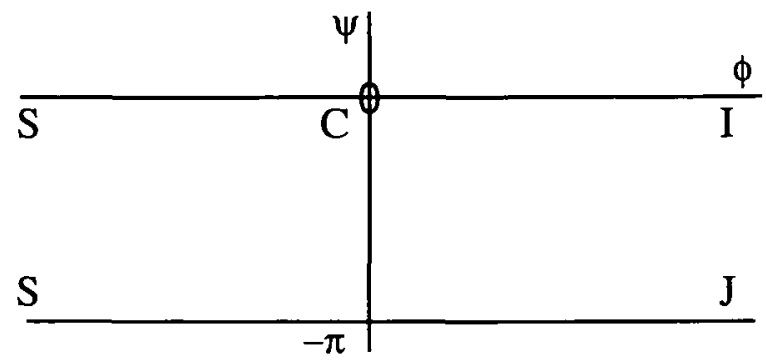

b
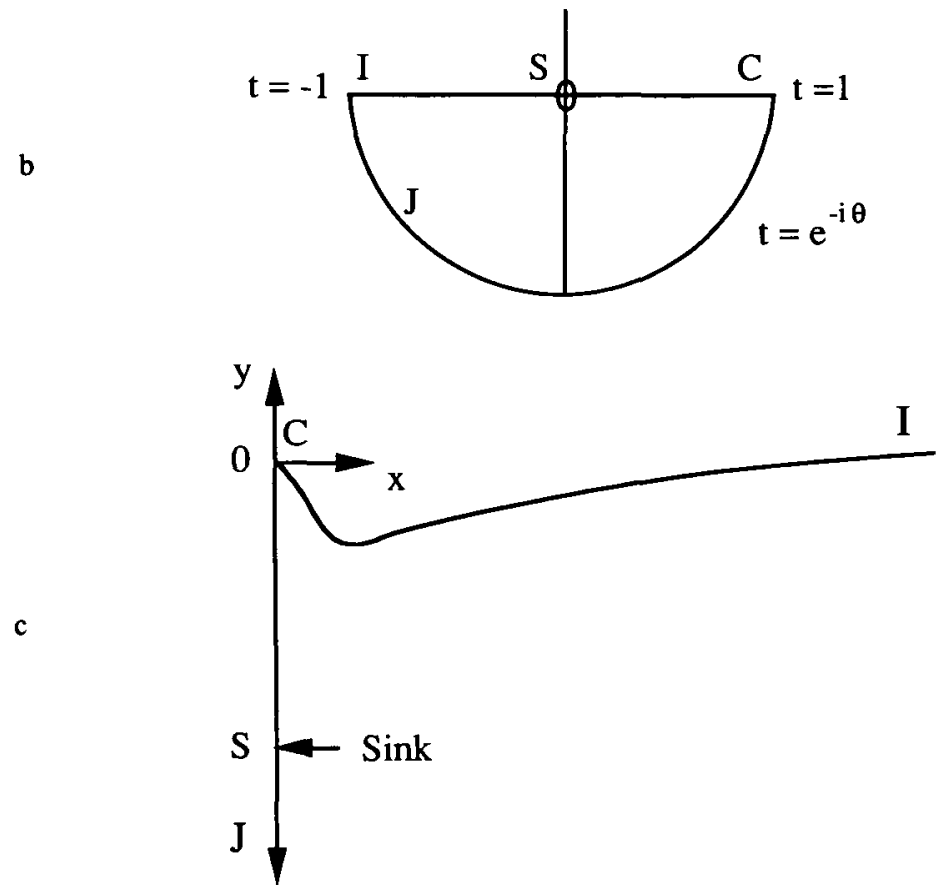

Figure 2. Mapped planes used in the problem formulation;

(a) the complex velocity potential $f$-plane,

(b) the lower half $t$-plane, and (c) the physical $z$-plane.

flow domain in the $z$-plane maps to the lower half of the unit circle in the $t$-plane, and the free surface thus corresponds to the boundary of the circle, i.e. $t=e^{-i \theta}, 0 \leq \theta \leq \pi$. The stream function $\psi$ is equal to zero on the free surface.

The magnitude of the velocity on the free surface is given by $\left|f^{\prime}(z)\right|$, which can be written as $\left|f^{\prime}(t)\right| /\left|z^{\prime}(t)\right|$ on $t=e^{-i \theta}, 0 \leq \theta \leq \pi$, and hence 
(4) becomes

$$
y(t)+\left|\frac{t-1}{t+1}\right|^{2}\left|\frac{d z}{d t}\right|^{2}=0,
$$

on $t=e^{-t \theta}, 0 \leq \theta \leq \pi$. Substituting for $d z / d t$ and writing in terms of $\theta$ we obtain, following Tuck and Vanden-Broeck [12],

$$
P\left(\theta ; b_{j}\right)=0, \quad 0 \leq \theta \leq \pi,
$$

where

$$
P\left(\theta ; b_{\jmath}\right)=Y(\theta)+\frac{4 \sin ^{2} \theta}{A^{2}(\theta)+B^{2}(\theta)},
$$

and

$$
\begin{aligned}
& Y(\theta)=\sum_{j=0}^{\infty} b_{j}\left[-\frac{1}{2} \cos j \theta-\frac{1}{2} \tan \frac{\theta}{2} \sin j \theta\right], \\
& A(\theta)=\sum_{j=0}^{\infty} b_{j}[(j-1) \cos j \theta+j \cos (j-1) \theta], \\
& B(\theta)=\sum_{j=0}^{\infty} b_{j}[(j-1) \sin j \theta+j \sin (j-1) \theta],
\end{aligned}
$$

and the problem reduces to that of finding the real values of $b$, which satisfy the condition that $P\left(\theta ; b_{j}\right)=0$.

To avoid numerical difficulties at $\theta=0$ and $\theta=\pi$, we investigate the limits as these values are approached. The condition that $P(\theta ; b)=$,0 at $\theta=\pi$ is satisfied if

$$
\sum_{j=0}^{\infty}(-1)^{j}(2 j-1) b_{j}=0 .
$$

To obtain a stagnation point at $\theta=0$, the condition

$$
\sum_{j=0}^{\infty} b_{j}=0
$$

which is equivalent to $z=0$ at $t=1$, must be satisfied.

The combination of (9), (10) and (11) gives the required conditions for the problem, and it remains to find the $b_{j}$. This can be done by truncating the infinite series to $N$ terms, $b_{1}, b_{2}, b_{2}, \ldots, b_{N}$. The Froude number is specified by the value of $b_{0}$ and hence $b_{0}$ has a known value. Requiring (10) and (11) to hold and (a) to hold at $\theta=j \pi /(N-1)$, for $j=1,2 \ldots N-$ 2 , gives $N$ nonlinear algebraic equations in $N$ unknowns, and solution of this system is equivalent to satisfying the condition of constant pressure on 
the free surface (4). Starting with guessed values for the $b_{j}$, a Newtonian iteration scheme can be used to solve the nonlinear systems.

\section{Results}

Tuck and Vanden-Broeck [12], in search of solutions with a stagnation point, found "the presence of short waves, which steepen as $F^{2}$ increases, and these solutions seem to be confined to $F^{2}<4$ ".

Using the series method, solutions without such waves were easily obtained for values of $0<F<1$ by computing solutions for small $N$ and then successively doubling $N$. In all cases, a set of coefficients which had given convergence at $N=120$, immediately gave convergence at $N=240,480, \ldots$. This means that with 120 coefficients the solution had converged at least to machine accuracy (14 figures in this case.) For larger values of the Froude number, solutions were obtained by successively incrementing $F$ and using results at the previous Froude number as a starting guess. In this manner, solutions up to and including $F=1.4$ were obtained. Solutions with $F<1.3$ were highly converged. For values of $F$ between 1.3 and 1.4 , no spurious numerical effects were obvious, either in the behaviour of the coefficients or the results, but the solutions were not so well converged. Near the top of this range, with $N=240$, numerical waves of the kind described by Tuck and Vanden-Broeck [12] began to appear, and these increased in amplitude until the failure of the numerical scheme at around $F=1.5$. Solutions at larger Froude numbers were not obtainable.

The results described above have been confirmed using an integral-equation formulation similar to that described in Forbes and Hocking [4]. The solutions obtained using this alternative formulation, with 240 discrete points approximating the integral, were found to exist for $0<F<1.4$, and once again waves began to appear near the top of this range of the Froude number, foreshadowing the breakdown of the solution procedure.

Figure 3 shows the free surface computed for the cases in which $F=0.5$, $F=1.0$ and $F=1.42$, the last being the largest at which the solutions were successfully calculated without any numerical waves present. In all cases the sink is situated at $y=-1.0$.

The trough situated at $x \simeq 1.0$ deepens as the Froude number increases, but there is nothing in the shape of the free surface which indicates physically why the breakdown should occur, such as a secondary stagnation point, as found in Forbes and Hocking [4], or the formation of a corner at the stagnation point, as suggested by Peregrine [11]. It is perhaps possible that the reasonably sudden failure of the numerical method at about $F=1.42$ 


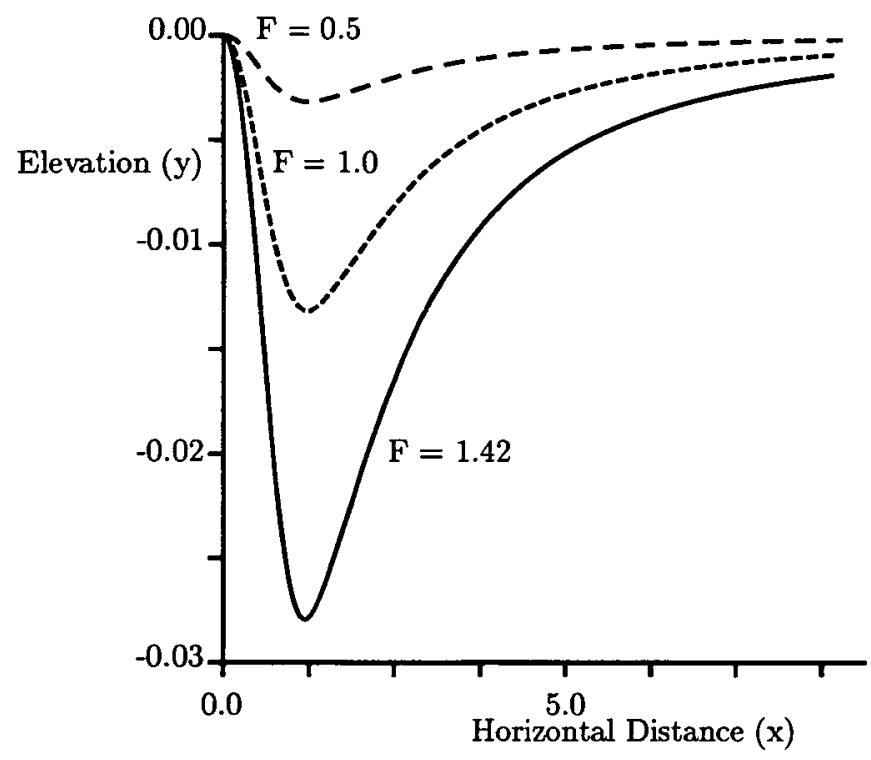

FIGURE 3. The shapes of the free surfaces computed with $F=0.5, F=1.0$ and $F=1.42$, the largest value of the Froude number at which steady flows of this type were obtained without numerical waves. The figure is scaled so that the sink is situated at $y=-1.0$.

could be due to the presence of a fold singularity in the nonlinear branch of solutions of this problem, similar to that observed by Vanden Broeck and Keller [13] (their Figure 4) for the solution type having a cusp at $x=0$. To test this hypothesis, we have written a modified version of the present algorithm, in which the first Fourier coefficient $b_{1}$ is specified, and the Froude number $F$ obtained numerically as part of the solution procedure. This modification should be capable of detecting a fold singularity if one is present near $F=1.42$, especially in view of the fact that the present spectral method should experience no difficulty converging even to the unstable solution branch anticipated beyond the fold. However, this modified algorithm did not detect any such fold, but likewise failed rather suddenly at a point corresponding to about $F=1.4$. We are therefore prepared to conjecture that the nonlinear branch of solutions is ultimately limited by a singularity at about $F=1.42$; its nature and physical significance are at present unknown.

A picture is emerging of the fluid behaviour as the sink is turned on and the Froude number increased. At low Froude numbers, flows with a stagnation point exist, and these persist until the Froude number approaches 1.4. No steady solutions appear to exist for $1.4<F<3.56$, suggesting the existence of some unsteady transition flow between the stagnation point flows and the single cusped solution at $F=3.56$ [12]. Figure 1 shows the difference in freesurface shape for the limiting stagnation-point flows and the cusp-like flows. 
For values of $F$ greater than 3.56 , the free surface is probably drawn down further toward the sink, meaning no solutions to the free-surface problem are possible, since the velocity at the sink must become infinite. When there are two layers of fluid present, however, flows in which the interface between the layers enters the sink directly are possible, and in this case, situations in which both fluids are drawn into the sink are probably realised.

\section{Summary}

The numerical results of the present paper lend strong support to the suggestion that steady solutions possessing a stagnation point at the free surface do indeed exist, in the approximate interval $0<F<1.4$. The results obtained by the Fourier-series method detailed here have been confirmed to a high degree of accuracy using an entirely different approach based on the use of an integral equation. For small Froude numbers, accurate results may be produced with a small number of Fourier coefficients, but near the limiting value $F=1.42$, it has been found necessary to employ a very great number of them in order to maintain accuracy. Some evidence for the presence of a limiting singularity at about $F=1.42$ has been described. It must, however, be admitted that there is controversy concerning the very existence of this type of solution, which our numerical results cannot entirely dispel. There is thus a clear need for a formal existence proof for this type of solution, which possesses a stagnation point at the free surface.

\section{Acknowledgements}

The authors would like to acknowledge the considerable assistance of E. O. Tuck in the preparation of this work. His suggestions, with those of a referee, resulted in significant improvement in the work finally presented. The series method used in this paper was developed by E. O. Tuck and J. M. Vanden Broeck. Part of this work was carried out while the first author was at the Centre for Water Research, University of Western Australia.

\section{References}

[1] M. Abramowitz and I. A. Stegun, (eds.), Handbook of mathematical functions (Dover, New York, 1970)

[2] A. Craya, "Theoretical research on the flow of nonhomogeneous fluids", La Houille Blanche 4 (1949), 44-45. 
[3] I. L. Collings, "Two infinite Froude number cusped free surface flows due to a submerged line source or sink" J. Aust. Math. Soc. Ser. B 28 (1986) 260-270.

[4] L. K. Forbes and G. C. Hocking, "Flow caused by a point sink in a fluid having a free surface", J. Aust. Math. Soc. Ser. B 32 (1990)231-250.

[5] G. C. Hocking, "Cusp-like free-surface flows due to a submerged source or sink in the presence of a flat or sloping bottom", J. Aust. Math. Soc. Ser. B 26 (1985) 470-486.

[6] G. C. Hocking, "Infinite Froude number solutions to the problem of a submerged source or sink", J. Aust. Math. Soc. Ser. B 29 (1988) 401-409.

[7] G. C. Hocking, "Critical withdrawal from a two-layer fluid through a line sink", J. Eng. Math. (1989) (in press).

[8] J. Imberger, "Selective withdrawal - a review", 2nd Int. Symposium on Stratified Fluids, Trondheim (1980) 381-394.

[9] A. C. King and M. I. G. Bloor, "A note on the free surface induced by a submerged source at infinite Froude number", J. Aust. Math. Soc. Ser. B 30 (1988) 147-156.

[10] H. Mekias and J. M. Vanden Broeck, "Supercritical free-surface flow with a stagnation point due to a submerged source", Phys. Fluids A 1 (10) (1989).

[11] D. H. Peregrine, "A line source beneath a free surface", Mathematics Research Center, Univ. Wisconsin Rept. 1248 (1972).

[12] E. O. Tuck and J. M. Vanden Broeck, "A cusp-like free-surface flow due to a submerged source or sink", J. Aust. Math. Soc. Ser. B 25 (1984) 443-450.

[13] J. M. Vanden Broeck and J. B. Keller, "Free surface flow due to a sink", J. Fluid Mech. 175 (1987) 109-117.

[14] J. M. Vanden Broeck, L. W. Schwartz and E. O. Tuck, "Divergent low-Froude number series expansion of non-linear free-surface flow problems", Proc. Roy. Soc. London Ser. $A 361$ (1978) 207-224.

[15] I. R. Wood and K. K. Lai, "Selective withdrawal from a two-layered fluid", J. Hyd. Res. 10 No. 4 (1972) $475-496$. 